\title{
Effect of model design, cushion construction, and interface pressure mats on interface pressure and immersion
}

\author{
Leigh Pipkin, MSPO; Stephen Sprigle, PhD, PT $^{*}$ \\ School of Applied Physiology, Georgia Institute of Technology, Atlanta, GA
}

\begin{abstract}
Measuring interface pressure (IP) is one way to characterize cushion performance in the clinic and laboratory. This study explored how the presence of four commercially available IP mats affected IP on and immersion of two buttocks models. We loaded seven cushions with each buttocks model and captured pressure data using FSA sensors (Vista Medical Ltd; Winnipeg, Manitoba, Canada). Analysis was performed to compare pressure magnitude and immersion. Overall, both pressure magnitude and immersion changed after mat introduction. A significant interaction existed between cushion and mat condition and cushion and model for all variables. Introducing an IP mat to the model-cushion interface alters the loading on the cushion. The mats bridged the contours of the model, causing a change in IP at the locations studied. Although immersion was statistically different between mat conditions, the magnitude of the difference was less than $1 \mathrm{~mm}$ once we accounted for the thickness of the mats. The significance of the cushion-mat interaction indicates that the mat effect differed across cushion design. Clinical and research users of pressure mats should consider the effect of mat presence, the effect of model design, and mat and buttocks interactions with cushions for successful use.
\end{abstract}

Key words: anatomic models, assistive technology, disability, interface pressure, pressure sensors, pressure ulcer prevention, rehabilitation, standards, test method, wheelchair seating, wheelchairs.

\section{INTRODUCTION}

Wheelchair cushions have been designed to reduce and distribute the mechanical forces applied to the skin and to decrease the chances of skin breakdown. Common designs use elastic foam, viscoelastic foam, elastomer, air, viscous fluid, or some combination thereof. A proper cushion should distribute pressure, minimize peak pressure at bony prominences, and encourage proper posture, as well as meet users' preferences in maintenance, comfort, and aesthetics [1]. With all of these factors in mind, a seating specialist or clinician may measure interface pressures (IPs) as one tool for selecting the optimal cushion [2].

To perform the measurement, a clinician places a pressure mat made up of an array of sensels between the client and the support surface. The pressure mapping software displays numerical values (often represented on a color scale) of IP at each sensel site. Clinicians can use these values, combined with other factors, to select wheelchair cushions for their clients.

For pressure mapping to be a useful clinical tool, the limitations of each system must be understood [3-4]. Several studies have reported on accuracy, creep, and hysteresis [5-11]. Environmental factors such as temperature and humidity have been observed to affect the accuracy of the sensors $[4,7,12]$. Other studies have found poor

Abbreviations: $\mathrm{HR}=$ high-resilience, ICC $=$ intraclass correlation coefficient, IP = interface pressure, ISO = International Organization for Standardization, IT = ischial tuberosity.

*Address all correspondence to Stephen Sprigle, PhD, PT; Georgia Institute of Technology, 490 Tenth St, NW, Atlanta, GA 30332-0156; 404-385-4302; fax: 404-894-9240.

Email: sprigle@gatech.edu

DOI: 10.1682/JRRD.2007.06.0089 
repeatability of some variables used to analyze IP data, especially around curved surfaces $[1,9,13]$. Peak pressure in particular is not recommended for use in analysis because of its lack of stability [1].

Additionally, the presence of pressure sensors may affect the environment and the pressure distribution being measured. One influence is hammocking, or bridging, support surface contours. In 1993, Ferguson-Pell and Cardi reported hammocking tests performed on the Tekscan seat system (both covered and uncovered) (Tekscan Inc; South Boston, Massachusetts), the Talley Pressure Monitor 3 (Talley Medical USA; Lansing, Michigan), and the FSA seat mat (Vista Medical Ltd; Winnipeg, Manitoba, Canada) with four cushions [6]. They used a buttocks-shaped loader gauge instrumented with three sensors to compare IP measurements taken with sensing mats at the modelcushion interface to those taken with no mat present. They found significant differences in IP for all cushions with the FSA and covered Tekscan mats present [6-7].

Our motivation for this study was to better understand the influences, if any, resulting from introducing a mat at the buttocks-cushion interface. IP measurement is a potentially useful tool that clinicians use to select cushions and researchers and manufacturers use to document cushion performance. With a fuller understanding of the mat effect, they can inform patients of proper use of these devices. Therefore, the objectives of this study were to explore changes in IP and immersion caused by addition of an IP mat using two buttocks models. The project tested the following hypotheses:

1. The presence of an IP mat will affect the IP measured between a buttocks model and wheelchair cushion.
2. The presence of an IP mat will change the immersion of a buttocks model when loaded on a wheelchair cushion.

3. The mats' effects on the cushion interface will differ across buttocks model designs.

\section{METHODS}

We used two buttocks models to collect data. These models were based on the anthropometry of a person with a $36 \mathrm{~cm}$ bitrochanteric breadth and $11 \mathrm{~cm}$ ischial spacing. These dimensions and the overall size and shape were consistent with the current International Organization for Standardization (ISO) buttocks models used for wheelchair testing [14]. One model was rigid and fabricated from wood (Figure 1), and the other was Elastack (Sutton Technologies Inc; North East, Maryland) thermoplastic, or gel, with embedded rigid cylinders to represent the ischial tuberosities (ITs) (Figure 2).

We collected pressure data at five points of interest (locations) along the central mediolateral axis of the models (Figures 1-2). The most inferior point of the model represented the location of the IT region of the human buttocks. The remaining sensors were located and named according to their vertical relationship to this inferior aspect. For example, the sensor located medial and $1 \mathrm{~cm}$ superior to the inferior point is labeled "medial" and the sensor located lateral and $1 \mathrm{~cm}$ superior is labeled "lateral."

We measured pressure with a custom-made FSA sensor array (Vista Medical Ltd; Winnipeg, Manitoba, Canada) consisting of 10 individual FSA sensors, with an
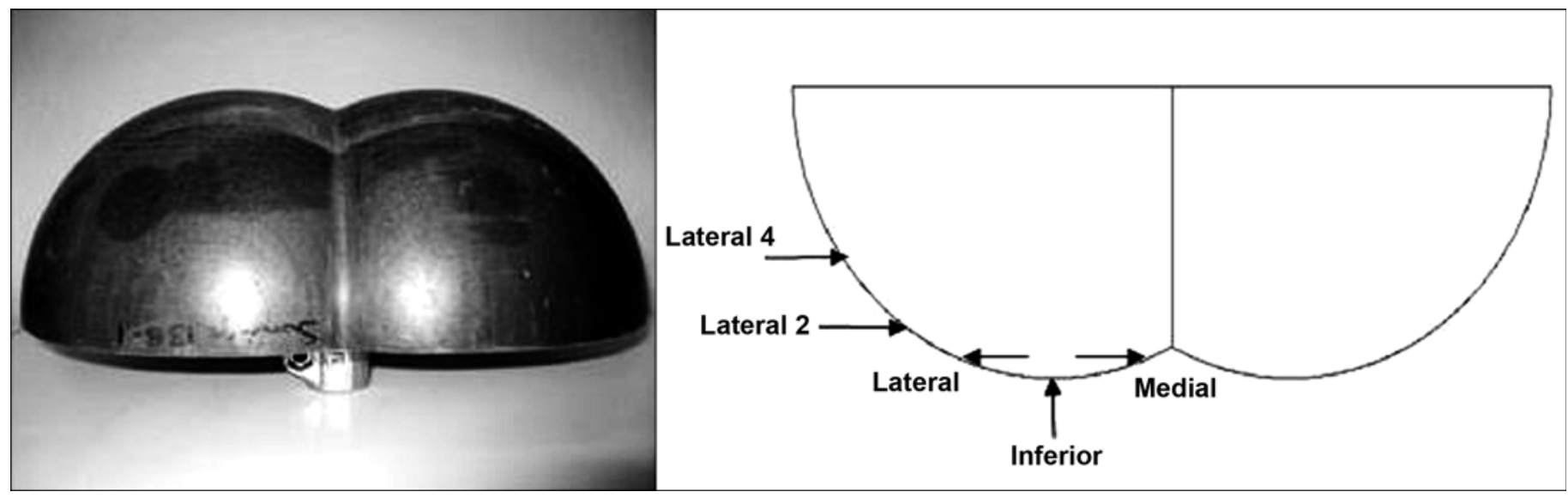

Figure 1.

Rigid buttocks model with sensor locations indicated. 


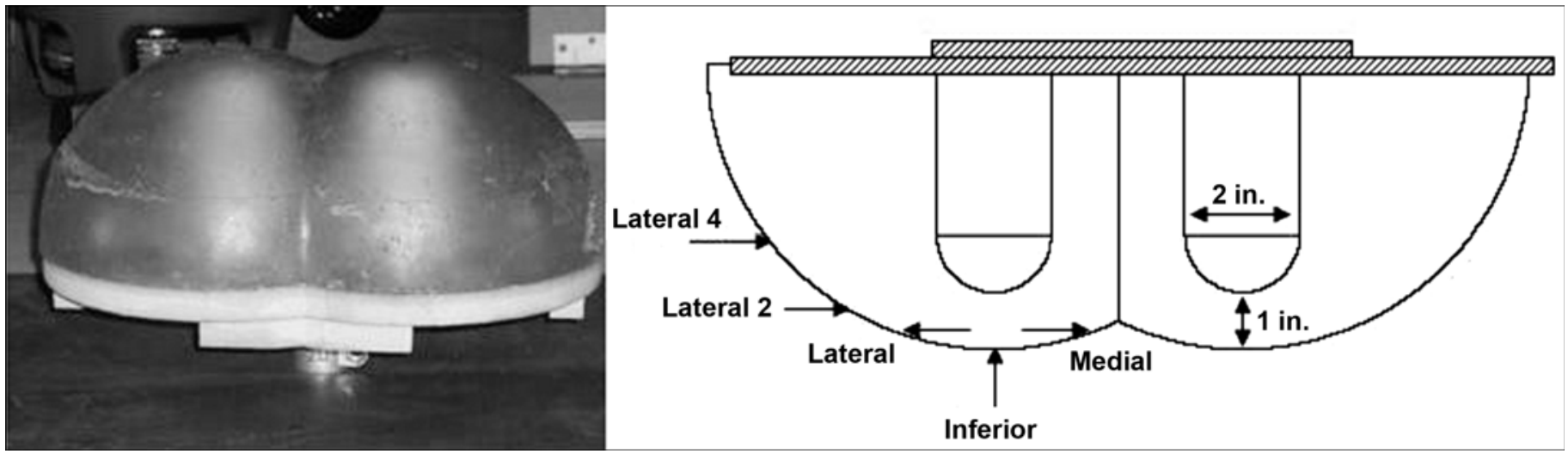

Figure 2.

Gel buttocks model with notations of sensor locations, diameter of embedded cylinder, and gel thickness under cylinders (Elastack, Sutton Technologies Inc; North East, Maryland).

active area of $1.96 \mathrm{~cm}^{2}$ each and a thickness of $0.6 \mathrm{~mm}$. We mounted two sensors at each of the five points to establish an active sensing area of $14 \times 28 \mathrm{~mm}$. An average of the two sensors was used as the data point for each location. The sensors were calibrated immediately before data were collected with the use of an air-filled bladder on the basis of recommended FSA protocol. Calibration was deemed acceptable if error at $100 \mathrm{mmHg}$ was less than 10 percent.

We then loaded buttocks models on seven cushions that were selected for their different designs and material construction (see Table 1 for names, manufacturers, and construction). Each cushion was loaded under five mat conditions: "no mat" and four commercially available IP mats placed atop the cushion being tested (see Table 2 for names, manufacturers, and characteristics). Cushions were adjusted according to manufacturers' instructions. Each model was fixed to a Zwick materials testing machine (Zwick USA LP; Kennesaw, Georgia). This device has a 0.2 percent load accuracy and $0.002 \mathrm{~mm}$ displacement accuracy. Cushions were placed such that the model was positioned $13 \mathrm{~cm}$ from the rear edge of the cushion. If the cushion had contour or site-specific loading areas, the model was positioned over this area. A $500 \mathrm{~N}$ load was selected to represent the upper-body weight of a $177 \mathrm{lb}$ human [14]. For each cushion and mat condition, a $550 \mathrm{~N}$ preload was applied for 120 seconds. After a 3-minute rest, the cushion was loaded to $500 \mathrm{~N}$ and held for 120 seconds. We captured IP values using the affixed FSA sensors and recorded height data from the Zwick. Three trials were taken with each cushion under each of the five mat conditions.

\section{Metrics}

We used test-retest comparisons to confirm variable repeatability using intraclass correlation coefficients (ICCs).

Table 1.

Cushions tested with buttocks models to measure interface pressures.

\begin{tabular}{lll}
\hline \multicolumn{1}{c}{ Cushion } & \multicolumn{1}{c}{ Distributor/Manufacturer } & \multicolumn{1}{c}{ Construction } \\
\hline Jay2 Deep Contour & Jay/Sunrise Medical (Longmont, Colorado) & $\begin{array}{l}\text { Viscous fluid and polyurethane foam in vinyl atop a nonde- } \\
\text { forming foam base }\end{array}$ \\
Action Xact Classic & Action Products Inc (Hagerstown, Maryland) & $9 / 16$ in. action cube pad and laminated foam base \\
Cloud & Otto Bock HealthCare (Minneapolis, Minnesota) & Viscous fluid packets of varying volume within a foam base \\
Star Standard Contour & Star Cushion Products (Freeburg, Illinois) & Single-valve adjustable air cushion \\
Tempermed & Tempur-Pedic (Lexington, Kentucky) & Viscoelastic foam \\
3 in. HR 45 Foam & Luxaire Cushion Corp (Newton Falls, Ohio) & Urethane foam with 45 IFD \\
3 in. HR 45 Foam, Segmented & Luxaire Cushion Corp & Urethane foam with 45 IFD, segmented into 2 $\times 2$ in. \\
& & squares extending 1 in. into 3 in. block \\
\hline HR = high-resilience, IFD = indentation force deflection. & \\
\hline
\end{tabular}


JRRD, Volume 45, Number 6, 2008

Table 2.

Characteristics of four interface pressure mats.

\begin{tabular}{|c|c|c|c|c|}
\hline Variable & Tekscan 5315 & CONFORMat & FSA & XSensor \\
\hline Model No. & 5315 & 5330 & UT1010 & Seat sensor pad \\
\hline Serial No. & $1227 \mathrm{~T} 1 / 4 / 3$ & 1445DT1 & 4437 & X361240 \\
\hline Sensor Type & Resistive & Resistive & Resistive & Capacitive \\
\hline Sensor Spacing (sensor/in. ${ }^{2}$ ) & 6.25 & 3.00 & 0.90 & 4.00 \\
\hline Sensor Configuration & 2,016 sensels in $42 \times 48$ array & 1,024 sensels in $32 \times 32$ array & 256 sensels in $16 \times 16$ array & 1,296 sensels in $36 \times 36$ array \\
\hline Mat Area $(\mathrm{L} \times \mathrm{W} \mathrm{cm})$ & $62 \times 53$ & $56 \times 63$ & $53 \times 53$ & $46 \times 46$ \\
\hline Overall Sensor Thickness (mm) & 0.48 & 1.34 & 2.00 & 1.00 \\
\hline Cover Material \& Thickness (mm) & Rip stop nylon (0.08) & Dartex (0.31) & Rip stop nylon (0.08) & Polyester (0.08) \\
\hline Sensor Material \& Thickness (mm) & $\begin{array}{l}\text { Mylar \& patented } \\
\text { semiconductive ink (0.32) }\end{array}$ & $\begin{array}{l}\text { Mylar \& patented } \\
\text { semiconductive ink (0.72) }\end{array}$ & $\begin{array}{l}1 \text { layer piezoresistive semiconductor } \\
(0.23), 2 \text { layers conductive fabric }(0.254)\end{array}$ & $\begin{array}{l}\text { Proprietary capacitive sensor } \\
(0.9)\end{array}$ \\
\hline Manufacturer & $\begin{array}{l}\text { Tekscan Inc; South Boston, } \\
\text { Massachusetts }\end{array}$ & $\begin{array}{l}\text { Tekscan Inc; South Boston, } \\
\text { Massachusetts }\end{array}$ & $\begin{array}{l}\text { Vista Medical Ltd; Winnipeg, Manitoba, } \\
\text { Canada }\end{array}$ & $\begin{array}{l}\text { XSENSOR Technology Corp; } \\
\text { Calgary, Alberta, Canada }\end{array}$ \\
\hline
\end{tabular}

Data were collected at all five sensor locations, and metrics reflecting magnitude and envelopment were investigated. Only metrics having ICC $\geq 0.9$ were used, and they were based on three sensor locations: lateral, inferior, and medial (Figures 1-2). Three metrics were calculated and grouped under two constructs: magnitude and immersion (Table 3).

\section{Magnitude}

Two metrics evaluated change in pressure magnitude: medial magnitude ratio and pressure sum ratio. We selected medial magnitude because this location approximates the area with the most complex curvature of the model. We calculated the pressure sum for each trial by adding together the pressures recorded at the three most inferior sensor locations. Collectively, these three locations represent the areas on the buttocks that transmit the most loading. When comparing mat effect, we calculated relative values by dividing the respective magnitude of the test cushion with that of the no-mat condition (Equations 1-2). When comparing the rigid and gel models, we considered only the no-mat condition, so magnitudes of pressure sum are reported without the normalization:

$$
\text { medial magnitude ratio }=\frac{(M)_{\text {test }}}{(M)_{\text {no_mat }}}
$$

and

$$
\text { pressure sum ratio }=\frac{[(M)+(I)+(L)]_{\text {test }}}{[(M)+(I)+(L)]_{\text {no_mat }}} \text {, }
$$

where $M=$ medial, $L=$ lateral, and $I=$ inferior.

\section{Immersion}

Immersion is a linear measure of how far a model sinks into a cushion. In this study, we measured immersion by the height (third metric) of the lowest part of the model above the test surface at the $500 \mathrm{~N}$ test load. The height is affected by the cushion thickness and the deformation of the cushion under load, and as a result, it varied widely across cushions. To account for this difference in cushion design, we characterized immersion by the height difference between the no-mat condition and the test conditions.

\section{Analysis}

As a measure of repeatability across trials, we calculated ICCs and used a two-factor analysis of variance to determine differences in magnitude and immersion across mat condition and cushion for each model. We then performed a single sample $t$-test to determine if the relative pressure magnitude metrics (medial magnitude and pressure sum ratios) were significantly different than 1.0, indicating a difference from the no-mat (reference) condition. Significance was determined at $p<0.05$.

Table 3.

Repeatability of metrics using intraclass correlation coefficients (ICCs) of gel and rigid buttocks models.

\begin{tabular}{llcc}
\hline \multirow{2}{*}{ Construct } & \multicolumn{1}{c}{ Metric } & \multicolumn{2}{c}{ ICC Model } \\
\cline { 3 - 4 } & & Gel & Rigid \\
\hline Magnitude & Pressure sum & 0.93 & 0.90 \\
& Medial magnitude & 0.91 & 0.94 \\
Immersion & Height & 1.00 & 1.00 \\
\hline \hline
\end{tabular}




\section{RESULTS}

\section{Repeatability}

All three variables had high test-retest reliability, with ICCs $>0.9$ for both buttocks models (Table 3). Peak pressure was found to be too volatile for use as a magnitude variable (ICC gel model: 0.61; ICC rigid model: 0.86), confirming a prior study [1].

\section{Magnitude}

Medial magnitude (pressure magnitude at the most medial site) dropped significantly after mat introduction for both buttocks models and across all mats (Table 4). Presence of the FSA and the Tekscan 5315 mats influenced medial magnitude more than the XSensor (XSENSOR Technology Corp; Calgary, Alberta, Canada) and CONFORMat (Tekscan Inc; South Boston, Massachusetts) as indicated by the lower ratios. Mat presence caused pressure sum to drop significantly when the rigid model was used, but when the gel model was used, pressure magnitude was not significantly different than the no-mat condition.

\section{Immersion}

buttocks model immersion into the cushions was significantly different across mat condition, cushion, and condition-cushion interaction for both models. Models immersed less under all-mat conditions, with changes ranging from 1 to $3 \mathrm{~mm}$ (Table 5).

Table 4.

Pressure magnitude results of four mats relative to no-mat condition of gel and rigid buttocks models.

\begin{tabular}{lccccc}
\hline \multirow{2}{*}{ Mat } & \multicolumn{2}{c}{ Pressure Sum } & & \multicolumn{2}{c}{ Medial Magnitude } \\
\cline { 2 - 3 } & Rigid* & Gel & & Rigid* & Gel $^{*}$ \\
\hline CONFORMat & 0.84 & $>0.99$ & & 0.86 & 0.88 \\
FSA & 0.61 & 1.02 & & 0.07 & 0.41 \\
XSensor & 0.78 & 1.01 & & 0.70 & 0.60 \\
Tekscan 5315 & 0.51 & 0.98 & & 0.06 & 0.40 \\
\hline Significantly different $(p<0.05)$. & & & \\
\hline \hline
\end{tabular}

Table 5.

Immersion of rigid and gel buttocks models across all cushions showing values significantly different from no-mat condition $(p<0.05)$.

\begin{tabular}{lcc}
\hline \multirow{2}{*}{ Mat } & \multicolumn{2}{c}{ Change in Height from No Mat (mm) } \\
\cline { 2 - 3 } & Rigid & Gel \\
\hline CONFORMat & 2.67 & 2.99 \\
FSA & 2.17 & 2.11 \\
XSensor & 1.17 & 1.99 \\
Tekscan 5315 & 1.26 & 1.81 \\
\hline \hline
\end{tabular}

\section{Cushion-Mat Interaction}

Significant interaction was found between cushion and mat condition in magnitude for both buttock models (Figure 3). The change in pressure magnitude differed depending on the cushion being tested. If a cushion-mat interaction did not exist, the differences from the no-mat condition would be similar across cushions for a given mat. In other words, the mat effect depends on the cushion being tested.

\section{Model Difference}

We assessed differences between buttocks models by comparing the data taken without an IP mat present. Pressure magnitude did not differ across models, whereas the gel model immersion was greater (lower model height) than the rigid model (Table 6).

\section{DISCUSSION}

\section{Mat Effect}

In general, the presence of the mats decreased pressure at the measured locations, indicating that the mats are redistributing the forces on the buttocks models. This result confirms the first hypothesis. When loaded, the mat attempts to take the shape of the indenter, but some folding or bending occurs. The result is a change in the areas at which load is borne. The capability to envelop the models is especially seen at the most medial sensor location, which represents the most complex curvature of

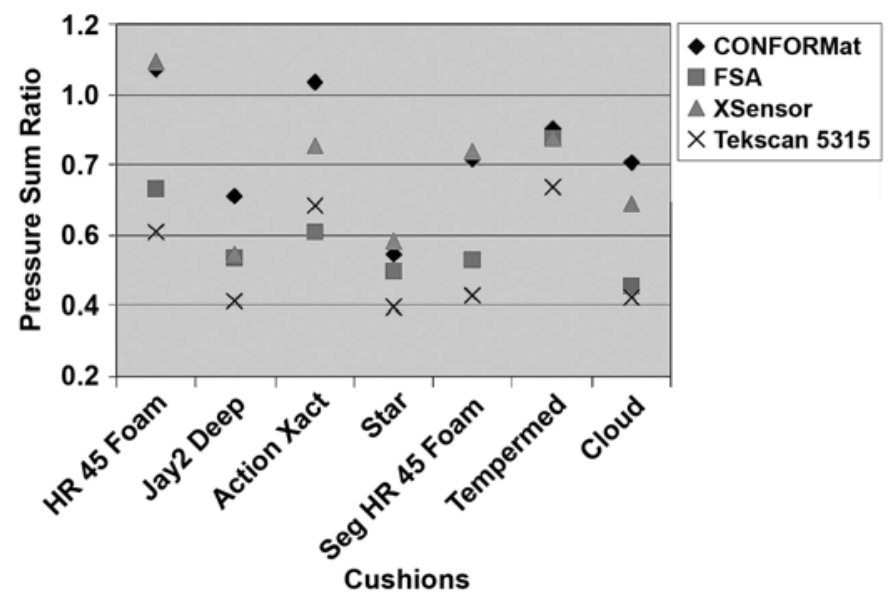

Figure 3.

Pressure sum ratios across cushion and mats illustrating interaction for rigid and gel buttocks models. HR = high-resilience, Seg = segmented. 
Table 6.

Overall difference in magnitude and immersion between rigid and gel buttocks models.

\begin{tabular}{lcc}
\hline Model & $\begin{array}{c}\text { Magnitude } \\
\text { (Pressure Sum } \\
\text { in mmHg) }\end{array}$ & $\begin{array}{c}\text { Immersion } \\
\text { (Height in mm) }\end{array}$ \\
\hline Rigid & 445 & 24.7 \\
Gel & 433 & 21.7 \\
\hline Difference & 12 & $3.0^{*}$ \\
\hline "Significantly different $(p<0.05)$. & \\
\hline \hline
\end{tabular}

the model and therefore the greatest challenge for the mats. All the mats bridge the contours and reduce cushion contact at this point. With the FSA and Tekscan 5315 mats, the pressure at the medial location dropped close to $0 \mathrm{mmHg}$ on some cushions, indicating minimal envelopment of the models at this point. The CONFORMat and the XSensor were better able to capture the load at this sensor location. Pressures at the three most inferior points (pressure sum) were significantly decreased on the rigid model, but no-mat effect was measured with the gel model.

Data also confirmed the second hypothesis that IP mats would affect immersion of the models. All mats significantly affected immersion, but this effect may not be clinically important. The maximum height change was $3 \mathrm{~mm}$ with the CONFORMat. This change in immersion was largely due to the nearly $2 \mathrm{~mm}$ thickness of the CONFORMat itself. Once the respective mat thicknesses are subtracted from the overall height changes, the models immersed about $1 \mathrm{~mm}$ less with the mats present than without. We do not believe this to be clinically significant, given this minimal change would not affect the contact area of the buttocks-cushion interface.

\section{Model-Cushion Deformation}

We used rigid and gel models to increase the generalizability of the results. Some studies have only used a gel model, although ISO currently uses only a rigid model. Incorporating results of rigid and nonrigid indenters permits assessment of the influence of indenter compliance on the mat effect. The deformation of the gel model under load appeared to allow the mats to conform in a manner that did not affect the total pressure on the inferior sensor locations (pressure sum) but still did affect the medial sensor values (Table 4). The gel model immersed slightly more than the rigid model, a result consistent with deformation of the gel model.
The cushion-mat interaction was significant for magnitude metrics (i.e., pressure sum and medial magnitude). This result means that the effect of using a mat varies across cushion design. Figure 3 illustrates this result. If no-mat effect existed, all values would be equal to 1 (equal to the no-mat condition). If no interaction existed, the individual mat effects would be similar across cushions. Note the spread of values for the high-resilience (HR) 45 foam contrasted against the minimal spread of the Tempermed cushion. The different mats affected the interface of the Tempermed much more similarly, whereas each respective mat had a different effect on the interface of the HR 45 foam.

\section{Limitations}

This study has several limitations that may affect the relevance of the study. Sensors were placed on the surface of the model and may potentially affect measurements. These sensors are designed as interface sensors and are $0.6 \mathrm{~mm}$ (0.02 in.) thick, so they may or may not affect results. Because mat characteristics are not fully described, changes in mat design or construction could alter the reported mat effect. The mats used in the study differed significantly in material construction and design. Despite those differences, their effect on IP was similar in several instances. Therefore, while we did not fully report material construction, the consistency in the results offers some assurance that minor changes will not fundamentally alter the finding that mat presence affects loading. Future studies should characterize physical properties of the mats to improve mat design. Finally, models of a single shape and size were used. While the design was based on human anthropometry and consistent with current testing methods, the results may or may not remain the same if different model shapes or sizes were used.

\section{CONCLUSIONS AND CLINICAL SIGNIFICANCE}

Adding an IP mat can change pressure magnitude, envelopment, and immersion. Within this study, effects varied with the type of mat and the type of cushion. Because of the mat effect, the pressures recorded by an IP mat may not reflect the actual buttocks-cushion IP. Because of the interaction between mat and cushion, the mat effect cannot be assumed constant across cushions for clinical comparison. 
These results have both research and clinical implications. Standardized test methods typically use buttocks models to evaluate cushions to achieve the necessary interlaboratory repeatability. The data indicate that indenter design can affect results and that models can significantly interact with cushion design. One cannot validly determine which model best reflects human subject measurements using the same metrics described here. Attaching individual sensors to human buttocks with a consistent location relationship would be difficult, if not impossible. Nonetheless, some model validation is needed to ensure that results in testing cushions with models reflect those found in testing cushions with human subjects.

The current ISO standard on wheelchair cushion performance does not include an IP test. The data that we report here indicate that the use of IP mats within standardized test methods might be problematic because of the interaction of the cushion and mat model. Therefore, these data can inform standards development. One approach that standard groups are investigating is to use instrumented buttocks models rather than IP mats to perform repeatable and consistent tests. The results of this study appear to support this approach.

Clinical implications are reflected by the changes in pressures due to mat introduction and by the differences in mat effects across cushions. The overall mat effect depressed IPs. Moreover, the interaction between cushion and mat means that mat effects differed by cushion model. In other words, mats interact with cushions differently. Translating this finding to the clinic suggests clinical IP measurements are not well suited for determining the "best" IP distribution across cushions. These mateffect results are consistent with our belief that clinical IP measurements can be used to "rule out" cushions rather than used as the singular way to select a cushion. If a clinician deems a certain IP to be unacceptable for a particular client, then he or she should not consider that cushion. Finally, the results corroborate our belief that any clinical assessment of a cushion must consider many factors to best meet the needs of the client.

\section{ACKNOWLEDGMENTS}

Leigh Pipkin is currently affiliated with Atlantic Prosthetics and Orthotics at UNC Hospitals, Chapel Hill, North Carolina.
This material was based on work supported by the Mobility Rehabilitation Engineering Research Center by way of funding from the National Institute on Disability and Rehabilitation Research, grant H133E030035.

Contents of this article do not necessarily represent the policy of the Department of Education or endorsement by the Federal Government.

The authors have declared that no competing interests exist.

\section{REFERENCES}

1. Sprigle S, Dunlop W, Press L. Reliability of bench tests of interface pressure. Assist Technol. 2003;15(1):49-57.

[PMID: 14760981]

2. Swain ID, Bader D. The measurement of interface pressure and its role in soft tissue breakdown. J Tissue Viability. 2002;12(4):132-46. [PMID: 12476502]

3. Shelton F, Lott JW. Conducting and interpreting interface pressure evaluations of clinical support surfaces. Geriatr Nurs. 2003;24(4):222-27. [PMID: 14560293]

4. Wolsley CJ, Hill PD. Review of interface pressure measurement to establish a protocol for their use in the assessment of patient support surfaces. J Tissue Viability. 2000;10(2): 53-57. [PMID: 11143638]

5. Allen V, Ryan DW, Lomax N, Murray A. Accuracy of interface pressure measurement systems. J Biomed Eng. 1993;15(4):344-48. [PMID: 8361161]

6. Ferguson-Pell MW, Cardi MD. Prototype development and comparative evaluation of wheelchair pressure mapping system. Assist Technol. 1993;5(2):78-91. [PMID: 10146318$]$

7. Ferguson-Pell MW, Cardi MD. Pressure mapping systems. Team Rehab Report. 1992;3(7):28-32.

8. Polliack AA, Craig DD, Sieh RC, Landsberger S, McNeal DR. Laboratory and clinical tests of a prototype pressure sensor for clinical assessment of prosthetic socket fit. Prosthet Orthot Int. 2002;26(1):23-24. [PMID: 12043923]

9. Polliack AA, Sieh RC, Craig DD, Landsberger S, McNeal DR, Ayyappa E. Scientific validation of two commercial pressure sensor systems for prosthetic socket fit. Prosthet Orthot Int. 2000;24(1):63-73. [PMID: 10855440]

10. Buis A, Convery P. Calibration problems encountered while monitoring stump/socket interface pressures with force sensing resistors: techniques adopted to minimise inaccuracies. Prosthet Orthot Int. 1997;21(3):179-82. [PMID: 9453089]

11. Nicholson G, Ferguson-Pell MW, Lennon P, Bain D. Comparative evaluation of pressure mapping systems: Bench testing results. Proceedings of the RESNA 2001 Annual Conference; 2001 Jun 22-26; Reno, Nevada. Arlington (VA): RESNA Press. p. 286-88. 
12. Ferguson-Pell MW. Design criteria for the measurement of pressure at the body/support interfaces. Eng Med. 1980; 9(4):209-14.

13. Sanders JE, Greve JM, Clinton C, Hafner BJ. Changes in interface pressure and stump shape over time: preliminary results from a trans-tibial amputee subject. Prosthet Orthot Int. 2000;24(2):163-68. [PMID: 11061203]
14. International Organization for Standardization (ISO). Wheelchair seating-Part 2: Determination of physical and mechanical characteristics of devices intended to manage tissue integrity —Seat cushions. Geneva (Switzerland): ISO; 2007. Report No.: ISO 16840-2:2007.

Submitted for publication June 19, 2007. Accepted in revised form February 4, 2008. 